\title{
Making nutrition guidelines fit for purpose
}

\section{Guidelines must ask the right questions and incorporate complexity to improve their relevance and quality, argue Lisa Bero and colleagues}

\author{
Lisa A Bero professor ${ }^{1}$, Susan L Norris scientist ${ }^{2}$, Mark A Lawrence professor ${ }^{3}$ \\ ${ }^{1}$ Charles Perkins Centre, Faculty of Medicine and Health, School of Pharmacy, University of Sydney, Sydney, Australia; ${ }^{2}$ Department of Information, \\ Evidence and Research, World Health Organization, CH-1211 Geneva, Switzerland; ${ }^{3}$ Institute for Physical Activity and Nutrition, School of Exercise \\ and Nutrition Science, Deakin University, Geelong, Australia
}

Dietary risk factors are the leading contributors to the global burden of disease. ${ }^{1}$ But what we choose to eat also affects the health of the planet. ${ }^{2}$ Nutrition guidance therefore serves multiple purposes, including promoting health and wellbeing, maintaining adequate nutrition, combating dietary excesses and imbalances associated with non-communicable diseases, and protecting sustainable food systems. However, existing nutritional guidelines often do not consider the right questions or fail to take full account of available evidence because they rely on methods borrowed from other fields. We need different types of evidence informed nutrition guidelines to tackle these unprecedented challenges to population health. ${ }^{3}$

\section{Unsuitability of current approaches}

The current approach to developing nutrition guidelines has been adapted from established methods for clinical practice guidelines and was not created with nutrition questions and evidence in mind. ${ }^{4}$ Although these methods are applicable to some types of nutrition guidelines, such as setting dietary or nutrient reference intake values, they are unsuitable for food and diet based guidelines for several reasons. ${ }^{5-7}$

Important questions related to nutrient interactions, dietary patterns, or food systems are studied mostly using observational designs rather than the randomised controlled trial that predominates in clinical studies. Randomised trials present several problems when studying nutrition exposures or interventions, ${ }^{89}$ although advances in trial design, such as pragmatic trials, can help improve problems related to generalisability of results. ${ }^{9}$

Rigorous guidelines are usually underpinned by a systematic review of available trials, and although review methods have also been evolving, they are optimised for randomised trials of single component interventions. Most of the nutrition guidelines and policy statements in the World Health Organization's e-Library of Evidence for Nutrition Actions (eLENA) database of systematic reviews and the Cochrane Library relate to single nutrient interventions, which can be evaluated by randomised trials. ${ }^{10}$ And the majority of nutrition systematic reviews in the Cochrane Library relate to evidence derived from studies of specific nutrients rather than of foods, dietary pattern, or food systems. ${ }^{11}$ As well as availability of evidence, selection of topics for systematic reviews may be affected by reviewers' interest in narrower, clinical topics.

A critical step to including observational studies in the systematic review process is assessing risks of bias. Tools for assessing human observational studies of exposure effects need further development. ${ }^{12}{ }^{13}$ A recent review of 62 tools for assessing observational studies of exposures could not recommend a specific tool but provided guidance for selecting one. ${ }^{14}$ Stakeholders from the nutrition field, including nutrition researchers and policy makers, should be involved in evaluating and achieving consensus on the most suitable tools for nutrition research.

The last crucial steps in guideline development are rating the certainty of the evidence and formulating recommendations based on the evidence and other considerations. Methods such as GRADE (Grading of Recommendations, Assessment, Development and Evaluation) are used infrequently, although guideline developers are starting to adopt this approach. ${ }^{4}$ But these methods typically rate randomised trials as higher quality than a body of evidence consisting of other study designs, which means the recommendations for broad nutritional interventions are likely to be less strong than those focused on narrow questions studied with randomised trials.

\section{Skewed evidence}

Because recommendations are based on a body of evidence, they are heavily influenced by the availability of research on particular topics, which, in turn, is influenced by funding 
sources. A recent scoping review examined original research and systematic reviews that assessed corporate influence on research agendas across different fields. ${ }^{15}$ The review found that industry tends to prioritise lines of inquiry that focus on products, processes, or activities that can be commercialised and marketed. For example, randomised trials sponsored by the food industry were more likely to test an intervention that manipulated a particular nutrient than trials with other sponsors. ${ }^{16}$ This could allow food companies to market manufactured products containing certain nutrients as beneficial to health. Additional studies in the scoping review analysed internal industry documents from the tobacco, alcohol, sugar, and mining industries. These highlighted the strategies used to reshape entire fields of research, ${ }^{15}$ including establishing research agendas that support industry policy positions, distract from research on harms of products, and protect industry from litigation.

Lack of transparency on funding sources makes it difficult to study differences between research with and without food industry sponsorship. ${ }^{17}$ But Coca-Cola makes sufficient information available on its funded projects to track its publications. Along with a focus on single nutrient research, over $40 \%$ of Coca-Cola funded projects focused on physical activity, suggesting that the company attempted to shift attention from the role of sugary drinks in obesity to the role of sedentary behaviour. $^{1819}$

Nutrition researchers ${ }^{1020-23}$ and organisations such as Cochrane ${ }^{11}$ are calling for reform of conventional evidence synthesis and translation approaches so they are better able to overcome limitations in available evidence and include evidence relevant to modern nutrition problems. Below we give two examples of reforms in public health.

\section{Incorporating complexity into systematic reviews}

Recent work on incorporating complexity into systematic reviews and guidelines will help to inform work in nutrition, particularly food and diet based guidelines. ${ }^{24}$ Taking a "complexity perspective" involves considering theory of change, causal pathways, complex systems properties, and context when conceptualising a review and when interpreting the evidence and formulating recommendations. ${ }^{25}$ Nutrition recommendations should be framed in a broader context than clinical recommendations, emphasising equity, human rights, and sociocultural acceptability as well as the benefits and harms of an intervention. ${ }^{26}$ Qualitative evidence can be synthesised to inform critical considerations such as acceptability and feasibility of complex interventions. ${ }^{27}$

Context is especially important when developing nutrition guidelines because of the diversity of purposes, exposure types, and intervention targets. The purpose of nutrition guidance extends across promoting health and wellbeing; nutritional adequacy for growth, maintenance, and repair; tackling dietary excesses and imbalances associated with non-communicable diseases and obesity; and protecting sustainable food systems. ${ }^{28}$ Nutrition exposure spans nutrients, foods, diets, and food systems. The intervention may target downstream, midstream, or upstream causes of nutrition problems. Thus, observational study designs that produce findings with high external validity are needed to account for the contextual characteristics of links between health and food and dietary patterns. ${ }^{29-31}$

\section{Advances in environmental health guidelines}

Environmental health science, which synthesises evidence to assess harms of environmental exposures, faces similar challenges to nutrition. ${ }^{32}$ Randomised trials of exposure to environmental risks are not available, and evidence syntheses must use data from observational human, animal, and in vitro mechanistic studies. In addition, environmental exposures are inherently complex and inter-related, making a randomised trial of a single chemical uninformative as well as unethical.

Environmental health researchers are innovating systematic review methods to evaluate and synthesise evidence from multiple data streams. ${ }^{32-35}$ Such integrative approaches may be useful for complex nutrition questions such as how evolutionary changes in animal diets affect health outcomes. ${ }^{36}$ GRADE is being modified for application to environmental health topics. ${ }^{37}$ For example, some frameworks for assessing research on environmental hazards start with an initial higher rating for observational studies than GRADE would apply. ${ }^{38}$ Importantly, the process for formulating the research questions is based on criteria such as priority and uncertainty rather than the availability of certain types of evidence or the methods needed to synthesise it. ${ }^{34}$

\section{Putting the horse before the cart}

The selection and evaluation of evidence for nutrition guidance are currently being driven by the methods for evidence synthesis rather than the questions that need to be answered. This is analogous to putting the cart before the horse; narrow questions studied with randomised trials are driving the development of guidelines rather than the complex nutrition problems that need to be solved to improve nutrition.

We believe this problem can be tackled by using theory and logic models to drive the development of nutrition guidelines. They can be used to strategically guide formulation of the research question(s), to hypothesise causal mechanisms, to identify the methods that best measure the hypothesised causal mechanism, and for evidence synthesis and translation. ${ }^{39}$

\section{Formulate the research question}

The first step in the development of nutrition guidance is to formulate research questions in relation to the type of nutrition exposure (nutrient, food, dietary pattern, food system, or interactions of these) or intervention target and health outcome. Research questions about risks or associations need to be differentiated from those about causation because they require different study designs. Questions related to nutrition exposure typically assess associations (eg, is energy intake associated with obesity?) whereas questions about interventions typically assess causality (eg, does menu labelling reduce food consumption?). These questions will then dictate the types of evidence that must be considered to answer the questions.

\section{Use theory to hypothesise causal mechanisms}

Building a logic model based on nutrition and health promotion science theory can help identify the causal mechanisms that need to be studied to answer a particular set of nutrition questions. For example, building a logic model to examine the question, "How can we improve the nutritional quality of the food supply and change consumer demand patterns to benefit population health, particularly obesity?" led to a series of 
systematic reviews of different strategies. ${ }^{39}$ Comparative studies of other species can also contribute theory for structuring research into the complex relationships between humans and food environments. ${ }^{36}$

Nutrition and health promotion science can provide theoretical insights to hypothesise the causal mechanism linking a nutrition exposure or intervention to a health outcome. Nutrition science theory helps explain the nature and scope of the associations between nutrients, foods, diets, food systems, and health outcomes ${ }^{40-44}$ and to model them. ${ }^{36}$ Health promotion science can help suggest causal mechanisms that link intervention types with health outcomes. Nutrition interventions can target downstream (treatment), midstream (prevention), or upstream (promotion) factors for a particular nutrition problem. For instance, interventions to reduce obesity could focus on gastroplasty (downstream), dietary behaviour (midstream), or social circumstances (upstream). Health promotion science theory can help explain the nature and scope of the relations between these nutrition interventions and health outcomes. Describing the potential causal relations can guide the development of questions to examine different parts of the model.

\section{Identify the type of evidence that should be included}

The questions developed from the logic model will then drive the selection of evidence for the systematic reviews. What constitutes the best evidence varies with the question being asked, and multiple types of evidence may be needed. ${ }^{45} 46$ The method selected to assess the hypothesised causal mechanism will be the one best able to account for the particular exposure type or intervention, as well as the contextual characteristics associated with the exposure or intervention. Narrow, nutrient specific questions can be examined with randomised trials whereas broader dietary pattern questions will usually require observational study designs. In addition, animal studies can provide mechanistic information or provide data on diet interactions with the environment. For example, nutritional ecology studies aim to understand how nutrition mediates the relations between animals and their environments, from short term homeostatic responses to longer term developmental and evolutionary adaptation. ${ }^{47}$

\section{Synthesise the evidence}

The challenge for guideline developers is to synthesise evidence from these different evidence streams, as sometimes done in environmental health. For example, to examine the effect of perfluorooctanoic acid on fetal growth systematic reviewers used predefined structured methods for integrating human and non-human evidence from different systematic reviews and linked these to describing the strength of the evidence. ${ }^{48}$

For public health guidelines examining broad questions for which the evidence comes from observational data, new ways of rating the evidence need to be considered that acknowledge factors such as generalisability and relevance for translation into practice. ${ }^{49} 50$ This means that observational studies might start out with a higher rating if they are the best fit for answering a question within the logic model. For example, a process such as GRADE could be modified to initially assign a "moderate" rather than "low" quality rating to human observational evidence, upgrading or downgrading based on predefined criteria relevant to the specific type of study. ${ }^{32}$

Other structured processes such as the Hill criteria, navigation guide, and US National Toxicology Program methods have also been proposed for rating the quality of diverse types of evidence. ${ }^{51}$ The main value of these approaches is that the judgments used to make recommendations are systematic and transparent.

\section{Influencing policy}

Systematic reviews are increasingly used to inform policy related to health, criminal justice, social welfare, and environmental risks. ${ }^{352}$ However, opposition to using systematic reviews is persistent and often led by commercial organisations. For example, drug companies have paid authors to criticise systematic review methods or challenge specific reviews and erroneously suggested that using evidence limits decisions and personal choice. ${ }^{5253}$ These criticisms can discourage policy makers from using systematic reviews of drug efficacy and safety to inform decisions. Mislabelling poorly conducted and methodologically unsound reviews as "systematic" also increases policy makers' distrust. ${ }^{54}$

Some countries now require systematic reviews to support claims about the health benefits of foods ${ }^{55}$ and for environmental risk assessments. ${ }^{51}$ Commercial interests then produce systematic reviews that meet regulatory requirements but not high methodological standards. ${ }^{54}$ The resistance to using systematic reviews as an evidence base for nutrition guidelines may be particularly hard to overcome because of the well documented industry influence on nutrition evidence. Systematic review methods may be unfamiliar to nutrition policy makers and the questions addressed by existing systematic reviews may not be relevant to them.

Our proposed approach to developing nutrition guidelines offers solutions to enhance the use of systematic reviews. Including policy makers in the production and prioritisation of systematic reviews can increase their uptake. ${ }^{52}$ Using theory and logic models to drive the development of nutrition guidelines is a way to incorporate policy makers' input early in the process of formulating questions and systematic reviews. Training policy makers, researchers, and nutrition scientists in systematic review methods can enable them to identify reviews that have high methodological standards. Lastly, transparency about industry influence in the production or evaluation of systematic reviews can enable policy makers to detect trustworthy reviews.

Although innovation is needed to fill some of the gaps in evidence synthesis methods, our approach offers opportunities for funders, researchers, and systematic reviewers to produce studies and synthesise evidence that can be used to inform optimal nutrition policy. Involving these stakeholders and policy makers in improving the methods for systematic reviews in nutrition will make the reviews underpinning nutrition guidelines more rigorous, transparent, usable, and relevant.

\section{Key messages}

Methods for developing clinical practice guidelines are not suitable for nutrition

The nutrition questions that need to be answered, rather than the availability of evidence, should drive nutrition guidance

Logic models and theory should be used to develop questions for review, identify and evaluate the most suitable types of study, and formulate recommendations

Innovation in evidence synthesis methods will be needed to assess properly the relevant evidence 
$\mathrm{SN}$ is an expert in guideline development and systematic review methods and has extensive experience at health systems, national, and global levels. ML is a public health nutritionist with extensive research experience in food policy, sustainable diets, and dietary guideline development. LB was invited by The BMJ to submit the paper and wrote the first draft. $S N$ and $M L$ made significant contributions to revisions of the paper. LB serves as guarantor.

Competing interests: We have read and understood BMJ policy on declaration of interest and declare that we have no competing interests. The authors alone are responsible for the views expressed in this article and they do not necessarily represent the views, decisions, or policies of the institutions with which they are affiliated.

Provenance and peer review: Commissioned; externally peer reviewed.

1 Afshin A, Sur PJ, Fay KA, etal . Health effects of dietary risks in 195 countries, 1990-2017: a systematic analysis for the Global Burden of Disease Study 2017. Lancet 2019. [Epub ahead of print.] 10.1016/S0140-6736(19)30041-8

2 Lawrence M, Burlingame B, Caraher M, Holdsworth M, Neff R, Timotijevic L. Public health nutrition and sustainability. Public Health Nutr 2015;18:2287-92.

10.1017/S1368980015002402 26344034

3 Burlingame B. Grand challenges in nutrition and environmental sustainability. Front Nutr 2014;1:3. 10.3389/fnut.2014.00003 25988107

4 Blake P, Durão S, Naude CE, Bero L. An analysis of methods used to synthesize evidence and grade recommendations in food-based dietary guidelines. Nutr Rev 2018;76:290-300 10.1093/nutrit/nux074 29425371

5 Blumberg J, Heaney RP, Huncharek M, etal . Evidence-based criteria in the nutritional context. Nutr Rev 2010;68:478-84. 10.1111/j.1753-4887.2010.00307.x 20646225

6 Dobrow MJ, Goel V, Upshur RE. Evidence-based health policy: context and utilisation Soc Sci Med 2004;58:207-17. 10.1016/S0277-9536(03)00166-7 14572932

7 Pawson R. Protocols, policy making and scientific progress. J Epidemiol Community Health 2012:66:386-7. 10.1136/jech-2012-201061 22473890

8 Naude CE, Durao S, Harper A, Volmink J. Scope and quality of Cochrane reviews of nutrition interventions: a cross-sectional study. Nutr J 2017;16:22 10.1186/s12937-017-0244-7 28388919

9 Schulze MB, Martínez-González MA, Fung TT, Lichtenstein AH, Forouhi NG. Food based dietary patterns and chronic disease prevention. BMJ 2018;361:k2396. 10.1136/bmj.k2396 29898951

10 Lawrence M, Wingrove K, Naude C, Durao S. Evidence synthesis and translation for nutrition interventions to combat micronutrient deficiencies with particular focus on food fortification. Nutrients 2016;8:555. 10.3390/nu8090555 27618094

11 Lawrence M, Naude C, Armstrong R, etal . A call to action to reshape evidence synthesis and use for nutrition policy[Editorial]. Cochrane Database Syst Rev 2016;11:ED000118.27883192

12 Bero L, Chartres N, Diong J, etal . The risk of bias in observational studies of exposures (ROBINS-E) tool: concerns arising from application to observational studies of exposures. Syst Rev 2018;7:242. 10.1186/s13643-018-0915-2 30577874

13 Morgan RL, Thayer KA, Santesso N, etal . Evaluation of the risk of bias in non-randomized studies of interventions (ROBINS-I) and the 'target experiment' concept in studies of exposures: Rationale and preliminary instrument development. Environ Int 2018;120:382-7. 10.1016/..envint.2018.08.018 30125855

14 Wang Z, Taylor K, Allman-Farinelli M, et al. A systematic review: tools for assessing methodological quality of human observational studies. Canberra, Australia: National Health and Medical Research Council (forthcoming)

15 Fabbri A, Lai A, Grundy Q, Bero LA. The influence of industry sponsorship on the research agenda: a scoping review. Am J Public Health 2018;108:e9-16. 10.2105/AJPH.2018.304677 30252531

16 Fabbri A, Chartres N, Scrinis G, Bero LA. Study sponsorship and the nutrition research agenda: analysis of randomized controlled trials included in systematic reviews of nutrition interventions to address obesity. Public Health Nutr 2017;20:1306-13. 10.1017/S1368980016003128 27989264

17 Fabbri A, Chartres N, Bero LA. Study sponsorship and the nutrition research agenda: analysis of cohort studies examining the association between nutrition and obesity. Public Health Nutr 2017;20:3193-9. 10.1017/S1368980017002178 28851466

18 Fabbri A, Holland TJ, Bero LA. Food industry sponsorship of academic research: investigating commercial bias in the research agenda. Public Health Nutr 2018;21:3422-30. $10.1017 /$ S1368980018002100 30157979

19 Rey-López JP, Gonzalez CA. Research partnerships between Coca-Cola and health organizations in Spain. Eur J Public Health 2018. 10.1093/eurpub/cky175 30169613

20 Cannon G, Leitzmann C. The new nutrition science project. Public Health Nutr 2005;8(6A):673-94. 10.1079/PHN2005819 16236201

21 Fardet A, Rock E. Toward a new philosophy of preventive nutrition: from a reductionist to a holistic paradigm to improve nutritional recommendations. Adv Nutr 2014;5:430-46. 10.3945/an.114.006122 25022992

22 Pelletier DL, Porter CM, Aarons GA, Wuehler SE, Neufeld LM. Expanding the frontiers of population nutrition research: new questions, new methods, and new approaches. $A d v$ Nutr 2013;4:92-114. 10.3945/an.112.003160 23319128

23 Penders B, Wolters A, Feskens EF, etal . Capable and credible? Challenging nutrition science. Eur J Nutr 2017;56:2009-12. 10.1007/s00394-017-1507-y 28718015

24 Norris SL, Rehfuess EA, Smith $\mathrm{H}$, etal . Complex health interventions in complex systems: improving the process and methods for evidence-informed health decisions. BMJ Glob Health 2019;4(Suppl 1):e000963. 10.1136/bmjgh-2018-000963 30775018
25 Petticrew M, Knai C, Thomas J, etal . Systematic reviews of complex systems for health decision-making: implications of a systems perspective for framing the question and for inclusion criteria. BMJ Global Health 2019;4:e00089910.1136/bmjgh-2018-000899.

26 Rehfuess EA, Stratil JM, Scheel IB, Portela A, Norris SL, Baltussen R. The WHO-INTEGRATE evidence to decision framework version 1.0: integrating WHO norms and values and a complexity perspective. BMJ Glob Health 2019;4(Suppl 1):e000844. 10.1136/bmigh-2018-000844 30775012

27 Booth A, Noyes J, Flemming K, etal . Formulating questions to address the acceptability and feasibility of complex interventions in qualitative evidence synthesis. BMJ Global Health [forthcoming].

28 Food and Agriculture Organization. Sustainable diets and biodiversity directions and solutions for policy, research and action. FAO, 2012.

29 Cartwright N. A philosopher's view of the long road from RCTs to effectiveness. Lancet 2011:377:1400-1. 10.1016/S0140-6736(11)60563-1 21520508

30 Luke DA, Stamatakis KA. Systems science methods in public health: dynamics, networks, and agents. Annu Rev Public Health 2012;33:357-76. 10.1146/annurev-publhealth-031210-101222 22224885

31 Temple NJ. How reliable are randomised controlled trials for studying the relationship between diet and disease? A narrative review. Br J Nutr 2016;116:381-9. 10.1017/S0007114516002129 27267302

32 Woodruff TJ, Sutton P. The Navigation Guide systematic review methodology: a rigorous and transparent method for translating environmental health science into better health outcomes. Environ Health Perspect 2014;122:1007-14. 10.1289/ehp.1307175 24968373

33 Rooney AA, Boyles AL, Wolfe MS, Bucher JR, Thayer KA. Systematic review and evidence integration for literature-based environmental health science assessments. Environ Health Perspect 2014:122:711-8. 10.1289/ehp.1307972 24755067

34 Vandenberg LN, Ågerstrand M, Beronius A, etal . A proposed framework for the systematic review and integrated assessment (SYRINA) of endocrine disrupting chemicals. Environ Health 2016;15:74. 10.1186/s12940-016-0156-6 27412149

35 Whaley P, Halsall C, Ågerstrand M, etal . Implementing systematic review techniques in chemical risk assessment: Challenges, opportunities and recommendations. Environ Int 2016:92-93:556-64. 10.1016/j.envint.2015.11.002 26687863

36 Raubenheimer D, Simpson SJ. Nutritional Ecology and Human Health. Annu Rev Nutr 2016;36:603-26. 10.1146/annurev-nutr-071715-051118 27296501

37 Morgan RL, Thayer KA, Bero L, etal . GRADE: Assessing the quality of evidence in environmental and occupational health. Environ Int 2016;92-93:611-6. 10.1016/i.envint.2016.01.004 26827182

38 Rooney AA, Cooper GS, Jahnke GD, etal . How credible are the study results? Evaluating and applying internal validity tools to literature-based assessments of environmental health hazards. Environ Int 2016;92-93:617-29. 10.1016/j.envint.2016.01.005 26857180

39 Anderson LM, Petticrew M, Rehfuess E, etal . Using logic models to capture complexity in systematic reviews. Res Synth Methods 2011;2:33-42. 10.1002/jrsm.32 26061598

40 Heaney RP. Nutrients, endpoints, and the problem of proof. J Nutr 2008;138:1591-5. 10.1093/jn/138.9.1591 18716155

41 Heaney RP. Nutrition, chronic disease, and the problem of proof. Am J Clin Nutr 2006;84:471-2. 10.1093/ajcn/84.3.471 16960157

42 Lappe JM, Heaney RP. Why randomized controlled trials of calcium and vitamin D sometimes fail. Dermatoendocrinol 2012;4:95-100. 10.4161/derm.19833 22928064

43 Heaney RP. Design and analysis of clinical trials of nutrients: author reply. Nutr Rev 2014;72:354-54. 10.1111/nure.12118 24738487

44 Stanton RA. Diet and nutrition: the folly of the reductionist approach. Med J Aust 2013;198:350-1. 10.5694/mja13.10297 23581941

45 Petticrew M, Roberts H. Evidence, hierarchies, and typologies: horses for courses. $J$ Epidemiol Community Health 2003;57:527-9. 10.1136/jech.57.7.527 12821702

46 Gough D, Elbourne D. Systematic research synthesis to inform policy, practice and democratic debate. Soc Policy Soc 2002;1:225-36. 10.1017/S147474640200307X

47 Raubenheimer D, Simpson SJ, Tait AH. Match and mismatch: conservation physiology, nutritional ecology and the timescales of biological adaptation. Philos Trans $R$ Soc Lond B Biol Sci 2012;367:1628-46. 10.1098/rstb.2012.0007 22566672

48 Lam J, Koustas E, Sutton P, etal . The navigation guide-evidence-based medicine meets environmental health: integration of animal and human evidence for PFOA effects on fetal growth. Environ Health Perspect 2014:122:1040-51. 10.1289/ehp.1307923 24968389

49 Green LW. Closing the chasm between research and practice: evidence of and for change. Health Promot J Austr 2014;25:25-9. . 10.1071/HE13101 24666557

50 Braveman PA, Egerter SA, Woolf SH, Marks JS. When do we know enough to recommend action on the social determinants of health?Am J Prev Med 2011;40(Suppl 1):S58-66. 10.1016/j.amepre.2010.09.026 21146780

51 National Research Council (U.S.). Board on Environmental Studies and Toxicology. Committee to Review the IRIS Process. Review of EPA's Integrated Risk Information System (IRIS) Process. National Academies Press, 2014

52 Fox DM. Evidence and health policy: using and regulating systematic reviews. Am J Public Health 2017;107:88-92. 10.2105/AJPH.2016.303485 27854522

53 Forsyth SR, Odierna DH, Krauth D, Bero LA. Conflicts of interest and critiques of the use of systematic reviews in policymaking: an analysis of opinion articles. Syst Rev 2014;3:122. 10.1186/2046-4053-3-122 25417178

54 Bero L. Systematic review: a method at risk for being corrupted. Am J Public Health 2017;107:93-6. 10.2105/AJPH.2016.303518 27854519

55 Australian Government. Australia New Zealand Food Standards Code: standard 1.2.7—nutrition, health and related claims. F2017C01048. 2017. https://www.legislation. gov.au/Details/F2018C00942

Published by the BMJ Publishing Group Limited. For permission to use (where not already granted under a licence) please go to http://group.bmj.com/group/rights-licensing/ permissions 